\title{
Introduction: A Special Issue on Frontiers of Electron Microscopy in Materials Science
}

This issue of Microscopy and Microanalysis contains contributions presented at the Frontiers of Electron Microscopy in Materials Science (FEMMS) meeting held in Kasteel Vaalsbroek, The Netherlands, on September 25-30, 2005. Tenth in the series of biennial conferences, the meeting focused on the latest developments in the field of advanced instrumentation and application of electron microscopy in materials science. The international character of this series of conferences was once again emphasized by the presence of over 140 delegates whose interests include academia, national laboratories, and industry from 16 countries representing all areas of the globe.

FEMMS 2005 covered a broad scope of topics ranging from the prospects for aberration correctors and monochromators in materials science to sophisticated demonstrations of microscopic investigations of lattice defects and internal boundaries in all types of structural and functional materials. The multitude of contributions thus represented a state-of-the-art overview of the microscopic techniques now being applied to solve some of the most relevant materials science problems. The articles collected in this issue range from fundamental treatments of the new electron optical components to demonstrations of advanced application of novel high resolution and analytical techniques.

The conference was organized by the Ernst Ruska-Centre for Microscopy and Spectroscopy with Electrons with major sponsorship by the Research Centre Jülich, RWTH Aachen University, and DFG, the German Research Foundation. Sponsors also included FEI Company, JEOL Ltd., Carl Zeiss Inc., Gatan Inc., E. A. Fischione Inc., Nion Co., Technoorg Linda Ltd., Soft Imaging System GmbH, EDAX AMETEK Inc., HWL Scientific Instruments, CEOS GmbH, BAL-TEC AG, NanoMEGAS, Hitachi HighTechnologies Europe GmbH, Thermo Electron Corp., and Oxford Instruments Plc. or their German branches. The conference organization was underpinned by the meticulous work carried out by Helene-Ingrid Rische-Radloff, Stefanie Stadler, and Gabriele Waßenhoven who deserve very special thanks.

While the FEMMS 2005 contributions referring to fundamental aspects of the technique are being published in this special issue of Microscopy and Microanalysis, manuscripts addressing materials science applications have been published in a recent issue of the Journal of Materials Science. All articles have been reviewed by two or more referees, and the editors are most grateful to the reviewers for their efficient work.

Knut Urban Joachim Mayer Martina Luysberg Karsten Tillmann 